\title{
Lesiones malignas de la piel en trabajadores del sector pesquero: Revisión sistemática
}

\section{Malignant Skin Neoplasms in Workers in the Fishing Industry: Systematic Review}

\author{
Beatriz Muñoz-Cobo-Orosa ${ }^{1}$ \\ Claudia Varela-Serrano ${ }^{1}$ \\ Mercedes Rodriguez-Ledott ${ }^{1}$ \\ Javier Sanz-Valero \\ Unidad Docente de Medicina del Trabajo Comunidad Autónoma del País Vasco, Vitoria, España. \\ Escuela Nacional de Medicina del Trabajo, Instituto Carlos III, Madrid, España.
}

Fechas · Dates

Recibido: 2020.10 .29

Aceptado: 2020.01.19

Publicado: 2021.01.21

\section{Correspondencia $\cdot$ Corresponding Author}

Mercedes Rodríguez Ledott.

Unidad Docente de Medicina del Trabajo del País Vasco.

Correo electrónico: mercheledott.r@gmail.com 


\title{
Resumen
}

Objetivo: Revisar la literatura científica relacionada con los efectos de la exposición ocupacional a factores de riesgo para neoplasias cutáneas en trabajadores del sector pesquero.

Método: Análisis crítico de los trabajos recuperados mediante revisión sistemática en MEDLINE (PubMed), EMBASE, The Cochrane Library, Scopus, Web of Science y Literatura Latinoamericana y del Caribe en Ciencias de la Salud (LILACS). Se evaluó la calidad de los artículos seleccionados mediante el cuestionario STROBE.

Resultados: Al atribuir los criterios de inclusión y exclusión a la búsqueda, se aceptaron 11 estudios para su revisión. En 7 de ellos, se obtuvo una asociación estadísticamente significativa entre neoplasia cutánea y exposición ocupacional.

Conclusiones: Se observa una relación entre lesiones malignas y exposición a radiación ultravioleta en el ámbito laboral, no obstante, la existencia de posibles sesgos hace que los resultados deban de tomarse con precaución.

Palabras clave: Neoplasias cutáneas; Enfermedades ocupacionales; Salud Laboral; Explotaciones Pesqueras; Industria pesquera.

\begin{abstract}
Objective: To review the scientific literature related to the effects of occupational exposure to risk factors for skin neoplasms in fishery workers.
\end{abstract}

Method: Critical analysis of the papers recovered through systematic review from en MEDLINE (PubMed), EMBASE, The Cochrane Library, Scopus, Web of Science y Literatura Latinoamericana y del Caribe en Ciencias de la Salud (LILACS). STOROBE guidelines were followed to evaluate their quality.

Results: After attributing inclusion and exclusion criteria to the search, 11 articles were accepted for review and critical analysis. In 7 of them, a statistically significant association was obtained between skin neoplasia and occupational exposure in fishermen.

Conclusions: Despite having found studies that were significant in terms of the relationship of malignant lesions and exposure to ultraviolet radiation in the workplace, some of them did not control possible biases, therefore the results should be taken with caution.

Keywords: Skin Neoplasms; Occupational Diseases; Occupational Health; Fisheries; Fishing Industry.

\section{Introducción}

La exposición ocupacional a la radiación solar es un importante factor de riesgo para la salud en el sector pesquero(1).Los pescadores presentan una amplia variedad de lesiones cutáneas que pueden ser producidas por los altos niveles de radiación ultravioleta (UV) y también por exposición a los hidrocarburos aromáticos policíclicos ${ }^{(2)}$. Los rayos UV son la primera causa de neoplasias cutáneas, habiéndose demostrado mediante evidencia biológica la relación directa entre una 
exposición excesiva y cáncer de piel, tanto tipo melanoma como no melanoma (NMSC), dado el daño que este tipo de radiación produce en el ADN celular.

El cáncer de piel tipo no melanoma es a su vez la neoplasia cutánea más frecuente en todo el mundo, representando un tercio de la incidencia global total de tumores. Comprende el carcinoma espinocelular (SCC), así como el basocelular (BCC)(1). El carcinoma espinocelular consiste en una proliferación maligna de los queratinocitos, un tipo de células en estrato espinoso de la epidermis (capa externa de la piel). Por su parte, el carcinoma basocelular, afecta a las células pluripotentes de la capa basal de la epidermis, y es además el cáncer de piel más frecuente. El principal factor de riesgo para ambos es la exposición crónica a la radiación ultravioleta, afectando sobre todo a personas de piel clara. El cáncer de labio a su vez pertenece al grupo de carcinoma espinocelular. El melanoma es una neoplasia derivada de los melanocitos, siendo uno de los tumores cutáneos más agresivos. La exposición solar es el principal factor de riesgo para los tumores mencionados, especialmente las quemaduras en la infancia, afectando más que una exposición crónica y mantenida a la luz solar ${ }^{(1)}$.

Los pescadores pasan mucho tiempo en los barcos, con pocas o ninguna posibilidad de protegerse frente a la citada radiación, cuyo efecto se ve además incrementado por las propiedades reflectantes del agua (efecto albedo). De hecho, ciertos estudios han demostrado que la exposición individual diaria de un trabajador del sector pesquero a los rayos UV es de entre 65 y 542 Julios $/ \mathrm{m}^{2}$ en las áreas descubiertas de su piel, superando esta medida el límite establecido. En total, un 43\% de las medidas diarias de radiación en pescadores resultaron muy por encima de la dosis eritémica diaria ocupacional (dosis de radiación UV efectiva que causa un enrojecimiento perceptible en la piel humana, siendo su valor 100 julios $\left./ \mathrm{m}^{2}\right)^{(3)}$.

Por ello, se consideró de interés realizar una revisión de la bibliografía con el objetivo principal de examinar la literatura científica existente sobre la exposición laboral a factores de riesgo para el desarrollo de neoplasias cutáneas en el sector de la pesca, a fin de identificar las diferentes exposiciones ocupacionales y, los tipos de lesión cancerosa derivados de las mismas en trabajadores de dicho sector.

\section{Métodos}

\section{Diseño}

Estudio descriptivo transversal y análisis crítico de los trabajos recuperados mediante revisión sistemática.

\section{Fuente de observación de los datos}

Los datos se obtuvieron mediante búsquedas en Internet, MEDLINE (PubMed), EMBASE, The Cochrane Library, Scopus, Web of Science y Literatura Latinoamericana y del Caribe en Ciencias de la Salud (LILACS). 


\section{Tratamiento de la información}

Para definir los términos de la búsqueda se consultó el Thesaurus desarrollado por la U.S. National Library of Medicine (Medical Subject Headings - MeSH), considerándose adecuados «Skin Neoplasms», «Occupational Diseases», «Fisheries» y «Fishing Industry» como descriptores y texto libre en título y resumen. La ecuación de búsqueda principal se desarrolló, con la utilización de conectores booleanos, para su empleo en la base de datos MEDLINE, vía PubMed. Esta misma estrategia se adaptó a las características del resto de bases de datos consultadas; ver Tabla $\mathbf{1}$.

La búsqueda se realizó desde la primera fecha disponible hasta diciembre del 2019 y se completó con el listado bibliográfico de los artículos incluidos en la revisión.

\section{Selección de estudios}

Se eligieron para su revisión los artículos que cumplieron los siguientes criterios de inclusión: ser artículos originales observacionales publicados en revista revisada por pares; y que existiera una relación causal entre "exposición en pesca" y "neoplasia de piel" (entendiéndose como la aparición de lesiones cutáneas malignas como consecuencia directa de la exposición motivada por su actividad laboral) en población adulta.

Al aplicar los criterios de exclusión, fueron eliminados aquellos artículos con idiomas diferentes a los establecidos (inglés o castellano) y/o no encontrados a texto completo. Las neoplasias aceptadas como lesiones cutáneas malignas fueron: el cáncer de piel tipo melanoma y el cáncer de piel tipo no melanoma, comprendiendo el carcinoma basocelular y el carcinoma espinocelular en algunas de sus diferentes localizaciones, como: labio, nariz, cabeza, cuello.

La selección de artículos pertinentes se realizó por los autores de la presente revisión. Para dar por válida la inclusión de los artículos, se estableció que la valoración de la concordancia entre las tres autoras (índice Kappa) debía ser superior a 0,70 (buena o muy buena concordancia) ${ }^{(4)}$. Siempre que se cumpliera esta condición, las posibles discordancias se solucionarían mediante la consulta del cuarto autor (JSV) y posterior consenso entre las autoras de la revisión.

\section{Evaluación de la calidad metodológica}

La calidad de los artículos seleccionados se valoró utilizando como apoyo las pautas para informar los estudios observacionales STROBE (STrengthening the Reporting of Observational studies in Epidemiology)(5), que contiene una lista de 22 puntos de control esenciales que deben describirse durante la publicación de estos documentos. Para cada artículo seleccionado, se asignó un punto por cada ítem presente (en caso de no ser aplicable, no puntuaba). Cuando un ítem estaba compuesto por varios puntos, estos se evaluaron de forma independiente, dándole el mismo valor a cada uno de ellos y, posteriormente, se realizó un promedio (siendo éste el resultado final de ese ítem), de tal forma que en ningún caso se pudiera superar la valoración total de un punto por ítem. 


\section{Extracción de los datos}

Para determinar la actualidad de los estudios, se calculó el semiperiodo de Burton-Kleber (mediana de la edad) y el índice de Price (porcentaje de artículos con edad inferior a los 5 años).

Los artículos se agruparon según las variables a estudio, con el fin de sistematizar y facilitar la comprensión de los resultados, considerando los siguientes datos: primer autor, tipo del estudio, periodo y país donde se realizó el estudio, agente(s) de exposición, población de estudio (trabajadores), tiempo de exposición, efecto estudiado y resultados principales.

Tabla 1: Estrategia de búsqueda adaptada a cada una de las bases de datos bibliográficas.

\begin{tabular}{ll}
\hline Base de datos & Ecuaciones de búsqueda \\
\hline MEDLINE & ("Skin Neoplasms"[Mesh] OR "Skin Neoplasms"[Title/Abstract] OR "cutaneous tumor"[Title/ \\
(Pubmed) & Abstract] OR "Cancer of Skin"[Title/Abstract] OR "cutaneous tumour"[Title/Abstract] OR \\
& "Skin Cancer"[Title/Abstract] OR "dermatoma" OR "Cancer of the Skin"[Title/Abstract] OR \\
& "Skin neoplasia"[Title/Abstract] OR "skin tumor"[Title/Abstract] OR "skin tumour"[Title/ \\
& Abstract]) AND ("Occupational Diseases"[Mesh] OR "Occupational Disease"[Title/Abstract] OR \\
& "worker disease"[Title/Abstract] OR "occupational disorder"[Title/Abstract] OR "occupational \\
& dysfunction"[Title/Abstract] OR "Occupational illness"[Title/Abstract] OR "professional \\
& disease[[Title/Abstract] OR "Occupational Exposure"[Mesh] OR "Occupational Exposure"[Title/ \\
& Abstract] OR "Work Exposure"[Title/Abstract]) AND ("Fisheries"[Mesh] OR "Fisheries"[Title/ \\
& Abstract] OR "Fishing Industry"[Title/Abstract] OR "Fish Flour Industry"[Title/Abstract] OR \\
& "Fishery worker"[Title/Abstract] OR "Fisheries"[Title/Abstract] OR "fisher"[Title/Abstract] OR \\
& "Fishery"[Title/Abstract] OR "Fish Farm"[Title/Abstract] OR "Fish Hatcheries"[Title/Abstract] OR \\
& "Fish Hatchery"[Title/Abstract] OR "watermen"[Title/Abstract] OR "Waterman"[Title/Abstract] \\
& OR "Seamen"[Title/Abstract] OR "Fishermen"[Title/Abstract] OR "Sailor"[Title/Abstract] OR \\
& "Seafarer"[Title/Abstract])
\end{tabular}

EMBASE ('fishery'/exp OR 'fisheries' OR 'fishery' OR 'sailor'/exp OR 'marine corps personnel' OR 'marine crew' OR 'marine recruit' OR 'mariner' OR 'naval crew' OR 'sailor' OR 'seaman' OR 'submarine crew' OR 'submarine sailor' OR 'yachtsman' OR 'pisciculture'/exp OR 'farming, fish' OR 'fish farming' OR 'pisciculture' OR 'fisherman'/exp) AND ('occupational disease'/exp OR 'agricultural workers ' diseases' OR 'deformation, professional' OR 'occupational disease' OR 'occupational diseases' OR 'occupational disorder' OR 'occupational dysfunction' OR 'occupational illness' OR 'professional deformation' OR 'professional disease' OR 'occupational exposure'/exp OR 'exposure, occupational' OR 'occupational exposure') AND ('skin tumor'/exp OR 'cutaneous tumor' OR 'cutaneous tumour' OR 'dermatoma' OR 'skin neoplasia' OR 'skin neoplasm' OR 'skin neoplasms' OR 'skin tumor' OR 'skin tumour' OR 'tumor, skin' OR 'tumour, skin')

Scopus y Web Of Science (ecuación de entrada)

\begin{abstract}
("Skin Neoplasms" OR "cutaneous tumor" OR "Cancer of Skin" OR "cutaneous tumour" OR "Skin Cancer" OR "dermatoma" OR "Cancer of the Skin" OR "skin neoplasia" OR "skin tumor" OR "skin tumour") AND ("Occupational Disease" OR "worker disease" OR "occupational disorder" OR "occupational dysfunction" OR "occupational illness" OR "professional disease" OR "Occupational Exposure" OR "Occupational Exposure" OR "Work Exposure") AND ("Fishing Industry" OR "Fish Flour Industry" OR "fishery worker" OR "Fisheries" OR "fisher" OR "Fishery" OR "Fish Farm" OR "Fish Hatcheries" OR "Fish Hatchery" OR "watermen" OR "Waterman" OR "Seamen" OR "Fishermen" OR "Sailor" OR "Seafarer")
\end{abstract}

("skin neoplasms" [Subject descriptor] OR "skin neoplasms" [Words]) AND ("occupational diseases" [Subject descriptor] OR "occupational exposure" [Subject descriptor] OR "occupational diseases" [Words] OR "occupational exposure" [Words]) AND ("fisheries" [Subject descriptor] OR "fisheries" [Words]) 


\section{Resultados y discusión}

Con los criterios de búsqueda descritos, se recuperaron 67 referencias: 15

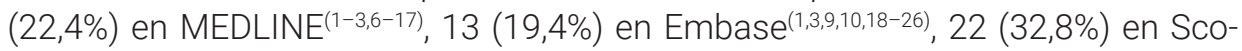
pus $^{(1-3,7-9,11,12,14,17,21,27-37)}$ y $17(25,4 \%)$ en Web of Science $e^{(1,3,6,7,9,10,13,22,28,29,38-44)}$. En la base bibliográfica LILACS no se recuperó ningún documento. Tras depurar los duplicados, aplicar los criterios de inclusión y exclusión y consultar los listados bibliográficos de los artículos escogidos (ver Figura 1), fue posible seleccionar 11 documentos para su revisión y análisis crítico (ver Tabla 2).

El acuerdo sobre la pertinencia de los estudios seleccionados entre las tres evaluadoras, calculado mediante el índice Kappa, fue del 71,4\% ( $p=0,01)$.

Los artículos elegidos presentaron una obsolescencia, según el Índice de Burton Kebler (IBK), igual a 24 años, con un Índice de Price (IP) del 18,18\%. Los años con mayor número de trabajos publicados fueron en el $2009^{(9,30)}$ y $1995^{(12,34)}$, con dos publicaciones respectivamente. La obsolescencia de los artículos relacionados fue mayor de lo esperado en el ámbito de las ciencias de la salud. Esto refleja el hecho de que la mayoría de estudios encontrados datan de una fecha de publicación anterior a los 10 años, habiendo sido publicados recientemente solo dos escritos, lo cual pone de manifiesto la necesidad de su actualización $n^{(4)}$.

Al aplicar el cuestionario STROBE, las puntuaciones oscilaron entre un mínimo de 12,32 (sobre 21 ítems) y un máximo de 16,33 (sobre 21 ítems), con mediana igual a 14,49 (ver Tabla 3). Ahora bien, la evaluación de la calidad de los estudios incluidos en este trabajo mediante STROBE resultó similar a la de otros artículos de revisión $(4,45)$.

Los trabajos revisados, fueron 8 estudios de cohortes $(7,9,12,13,29,30,34,36), 1$ estudio de casos y controles ${ }^{(1)}, 1$ estudio transversal(14) y 1 estudio de casos y controles y cohortes $^{(16)}$. De estos trabajos, 3 únicamente en Suecia ${ }^{(13,30,34)}, 2$ tanto en Suecia cómo en Finlandia, Islandia y Noruega(7,9) y 1 en Italia( ${ }^{(1)}$, Islandia(12), los EE.UU.(14), Finlandia ${ }^{(29)}$, Reino Unido ${ }^{(36)}$ y Canadá(16), todos ellos redactados en inglés.

La edad de la población incluida siempre fue superior a los 15 años, y el trabajo que presentó menor tamaño muestral fue Larese Filon et al(1), de $2019(n=126)$, siendo por el contrario el estudio que más personas incluyó el de Pukkala et al(9), de 2009 ( $n=15$ millones), sin diferenciar los diferentes tipos de neoplasias, por lo que no pudo obtenerse la población correspondiente a neoplasias cutáneas, lo que pudo suponer una sobreestimación en la síntesis cualitativa.

La población estudiada en cinco de los once trabajos (45,5\%) estuvo formada exclusivamente por hombres $(9,13,14,16,34)$, y en cuatro de los estudios $(36,4 \%)$, fueron incluidos individuos de ambos sexos ${ }^{(1,29,30,36)}$. En el resto, no constaba la distribución por sexos.

El agente de exposición mencionado en la gran parte de los estudios fue la radiación ultravioleta. Esto se debió a que casi la totalidad de los integrantes del sector pesquero incluidos en los estudios seleccionados trabajaban en el exterior y, en consecuencia, estaban expuestos a dicho factor de riesgo. Sin embargo, los estu- 
dios de Mikoczy ${ }^{(30)}$ y Svensson et al(34), analizaron también el efecto de la exposición a productos químicos, como dioxinas y furanos. Por su parte, los trabajos de Pukkala ${ }^{(9)}$, Rafnsson ${ }^{(12)}$ y Whitaker ${ }^{(36)}$ estudiaron la exposición al tabaco.

Durante la búsqueda se encontraron estudios observacionales en trabajadores del sector pesquero. En algunos artículos resultó difícil estimar si las lesiones malignas podrían atribuirse o no a exposición ocupacional, al tratarse de factores de riesgo existentes fuera del entorno laboral (radiación ultravioleta, productos químicos, tabaco). Alfonso et al, concluyeron que la variación ocupacional del riesgo relativo del carcinoma espinocelular podría asociarse a factores socioeconómicos $y$, en cierta medida, a exposiciones profesionales ${ }^{(7)}$. Burke et al, alegaron que los pescadores están expuestos a diversos factores, siendo uno de ellos la radiación ultravioleta ${ }^{(2)}$.Larese et al, elaboraron un estudio univariante, encontrando una asociación entre la profesión y las neoplasias de tipo espinocelular. Sin embargo, al abordar el estudio mediante análisis multivariante (de mayor evidencia estadística), los resultados se vieron modificados, obteniéndose falta de asociación. Esto pudo deberse a la escasa muestra del estudio(1).

El periodo de seguimiento de los estudios incluidos en esta revisión osciló entre un mínimo de 11 meses (Vitasa et a/(14)) y un máximo de 44 años (Alfonso et al(7)).

En los estudios seleccionados se analizó la asociación entre neoplasias de piel de tipo melanoma, tipo no melanoma (carcinoma espinocelular (SCC) y basocelular (BCC)) y cáncer de labio.

En siete de los once estudios (63,6\%) se demostró una relación estadísticamente significativa entre el desarrollo de cáncer de tipo no melanoma y el sector pesquero $^{(1,7,13,14,29,30,34)}$. Larese Filon et al, obtuvieron una OR = 4,3 (IC 95\% 1,1-16,4) mediante análisis univariante (muestra demasiado pequeña) en trabajadores pesqueros de origen italiano(1). En el trabajo de Alfonso et al, la razón de incidencia estandarizada para el carcinoma espinocelular en marineros (seamen) y pescadores (fishermen) mayores de 50 años de Finlandia, Islandia, Noruega y Suecia resultó significativo ( $\mathrm{SIR}=1,23$ IC 95\% 1,14-1,32; SIR= 1,47 IC 95\% 1,25-1,71, respectivamente). En participantes de entre 30 y 49 años no fue significativo(7). Pukkala et al, 1996, mostraron que la exposición acumulativa a radiación ultravioleta duplicaba el riesgo de cáncer de piel de tipo no melanoma entre pescadores finlandeses que llevasen más de 10 años trabajados ( $\mathrm{SIR}=1,8, \mathrm{IC}$ 1,2-2,5), y aumentaba proporcionalmente a los años trabajados ${ }^{(29)}$. El riesgo de cáncer espinocelular en pescadores suecos aumentó en los artículos de Svensson et al, (SIR=2,3 IC 95\% 1,5-3,5 en pescadores de la costa este; IRR 1,9 IC 95\% 1,2-3,1 en costa oeste)(34) y de Hagmar et al, (SMR= 2,79 IC 95\% 1,73-4,36)(13). Mikoczy et al, estudiaron y compararon la incidencia de cáncer en pescadores de la costa oeste y este de Suecia, y el carcinoma espinocelular resultó ser significativo sólo en los pescadores de la costa oeste (SIR=1,3 IC 95\% 1,08-1,55) (30). Vitasa et al, realizaron una regresión logística y demostraron que la edad avanzada, la presencia de pecas en la infancia y el tener ojos azules incrementaban el riesgo de los 3 tipos de lesiones neoplásicas estudiadas (SCC, BCC y queratosis actínica (AK)) en pescadores de la costa este de Maryland. La susceptibilidad para quemaduras solares estaba en relación con el 
BCC y AK, pero no con SCC. Marineros con una exposición acumulativa del cuartil superior presentaban 2,5 veces mayor riesgo de desarrollar SCC, comparado con el resto de cuartiles. Para el desarrollo de AK, el riesgo era 1,5 veces mayor en aquellos que superaban la media(14).

En cinco de los cinco trabajos que estudiaron el desarrollo del cáncer de labio en el sector pesquero se obtuvo una correlación positiva y estadísticamente significativa $(9,13,16,30,34)$.

La asociación entre el cáncer de piel de tipo melanoma y el sector pesquero resultó estadísticamente significativa en uno(29) de los cinco trabajos ${ }^{(9,12,29,30,34)}$ que estudiaron esta patología. Pukkala et al, 2009 relataron que los pescadores y los forestales eran los grupos con menor riesgo de melanoma maligno (SIR 0,51; IC $95 \%$ 0,45-0,58)(9). Sin embargo, en el artículo de Pukkala del año 1996, se puso de manifiesto que las quemaduras solares repetidas incrementaban el riesgo de melanoma en menores de 30 (SIR en trabajadores de cubierta y motor $=4,3$; IC $95 \% 3,1-6,5)^{(29)}$.

Cuatro de los once estudios incluidos no obtuvieron resultados estadísticamente

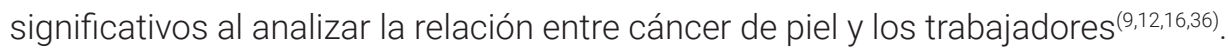

Todos los artículos encontrados relacionaron las lesiones malignas en los pescadores, en mayor o en menor medida, con la exposición laboral a la radiación ultravioleta, siendo los carcinomas basocelulares, espinocelulares y melanoma las lesiones más frecuentes. Rafnsson et al, asociaron otros tipos de cánceres a la exposición a radiación ultravioleta, asbesto y tabaco. Estos tipos de cáncer fueron: adenocarcinoma de estómago, adenocarcinoma de laringe, lengua, leucemia y adenocarcinoma de recto(12). La zona en la cual asentaron con mayor frecuencia las neoplasias dermatológicas descritas fue en el labio(7,9,13,14,16,30). Ranfnsson et al, ${ }^{(12)}$ Spitzer et al,$^{(16)}$ Pukkala et a $\left(^{(9)}\right.$, describieron otras zonas donde podrían producirse las lesiones malignas (cabeza, cuello y nariz). De todos los artículos revisados, sólo en tres no consta el tiempo de exposición al agente causante de neoplasias ${ }^{(1,7,9)}$. En la mayoría de artículos se demostró una relación lineal entre las citadas lesiones y la exposición a radiación ultravioleta.

Larese et al, evidenciaron que en Italia el bronceado era considerado positivo, por lo que la mayoría de individuos que trabajaban al aire libre no utilizaban medios de protección frente a la radiación solar (casco, gafas, crema protectora), siendo su exposición superior a la esperable, y, por ello, los resultados de su análisis pudieron verse sobreestimados ${ }^{(1)}$. Por el contrario el estudio realizado por Rafnsson et al, en Islandia, podría haber sido no significativo debido a la latitud nórdica y, por ende, a la menor exposición solar ${ }^{(12)}$. 
Tabla 2: Características y resultados principales de los estudios seleccionados en la revisión sobre las lesiones malignas de la piel en trabajadores del sector pesquero.

\begin{tabular}{|c|c|c|c|c|c|c|c|}
\hline Autor & $\begin{array}{l}\text { Tipo de } \\
\text { estudio }\end{array}$ & Periodo y país & $\begin{array}{l}\text { Agente(s) } \\
\text { (exposición) }\end{array}$ & $\begin{array}{l}\text { Población } \\
\text { (trabajadores) }\end{array}$ & $\begin{array}{l}\text { Tiempo } \\
\text { exposi- } \\
\text { ción }\end{array}$ & $\begin{array}{l}\text { Efecto } \\
\text { estudiado } \\
\text { (neopla- } \\
\text { sia) }\end{array}$ & Resultados principales \\
\hline $\begin{array}{l}\text { Larese } \\
\text { Filon et al, } \\
2019(1)\end{array}$ & $\begin{array}{l}\text { Casos y } \\
\text { controles }\end{array}$ & $\begin{array}{l}\text { Enero 2015-Di- } \\
\text { ciembre 2016, } \\
\text { Italia }\end{array}$ & -Radiación UV & $\begin{array}{l}\text { Casos } n=126 \\
H / M=1.17 \\
\text { Edad } M=72 \\
\text { Controles } \\
n=187 \\
H / M=0.35 \\
\text { Edad } M=72\end{array}$ & $\begin{array}{l}\text { No } \\
\text { consta }\end{array}$ & $\begin{array}{l}\text { NMSC } \\
\text { (BCC, SCC) } \\
\text { Carcinoma } \\
\text { basocelular } \\
\text { Carcinoma } \\
\text { escamoce- } \\
\text { lular }\end{array}$ & $\begin{array}{l}\text { Mayor riesgo entre profesión y } \\
\text { NMSC en análisis univariante. } \\
\mathrm{OR}=4,3(1,1-16,4) p=0,035\end{array}$ \\
\hline $\begin{array}{l}\text { Alfonso et } \\
\text { al, 2016(7) }\end{array}$ & $\begin{array}{l}\text { Cohorte } \\
\text { prospectiva }\end{array}$ & $\begin{array}{l}\text { 1961-2005 } \\
\text { Finlandia, Islan- } \\
\text { dia, Noruega, } \\
\text { Suecia }\end{array}$ & $\begin{array}{l}\text {-Radiación UV } \\
\text { - Hidrocarburos } \\
\text { policíclicos } \\
\text { aromáticos }\end{array}$ & $\begin{array}{l}n=12.9 \text { mi- } \\
\text { llones } \\
\text { H/M= no } \\
\text { consta } \\
\text { Edad: nacidos } \\
\text { entre } 1896 \text { y } \\
1960\end{array}$ & $\begin{array}{l}\text { No } \\
\text { consta }\end{array}$ & SCC & $\begin{array}{l}\text { SCC: SIR= 1,23 IC } 95 \% 1,14- \\
\text { 1,32 en marineros; SIR= 1,47 IC } \\
95 \% 1,25-1,71 \text { en pescadores } \\
\text { mayores de } 50 \text { años. }\end{array}$ \\
\hline $\begin{array}{l}\text { Mikoczy } \\
\text { et al, } \\
2009(30)\end{array}$ & $\begin{array}{l}\text { Cohortes } \\
\text { retrospec- } \\
\text { tivo }\end{array}$ & $\begin{array}{l}\text { 1965-2002, } \\
\text { Suecia }\end{array}$ & $\begin{array}{l}-\mathrm{N}-3 \text { PUFA } \\
\text {-PCBS } \\
\text {-Dioxinas } \\
\text {-UV-B }\end{array}$ & $\begin{array}{l}\mathrm{n}=20184 \\
\mathrm{H} / \mathrm{M}=1,32 \\
\text { Edad M: no } \\
\text { consta }\end{array}$ & $\geq 1$ año & $\begin{array}{l}\text { SCC } \\
\text { Melanoma } \\
\text { Carcinoma } \\
\text { de labio }\end{array}$ & $\begin{array}{l}\text {-SCC: SIR costa oeste } 1,3 \\
(1,08-1,55), \text { costa este } 1,36 \\
(0,92-1,93) \text {. } \\
\text {-Melanoma: SIR costa oeste } \\
1,14(0,85-1,5), \text { costa este } 0,28 \\
(0,08-0,71) \\
\text {-Ca labio: SIR costa oeste } 2,72 \\
(1,97-3,66), \text { costa este } 4,44 \\
(2,48-7,32)\end{array}$ \\
\hline $\begin{array}{l}\text { Pukkala } \\
\text { et al } \\
2009(9)\end{array}$ & Cohorte & $\begin{array}{l}\text { 1970, Dina- } \\
\text { marca } \\
\text { 1970-1980- } \\
\text { 1990, Finlandia } \\
\text { 1981, Islandia } \\
\text { 1960-1970- } \\
\text { 1980, Noruega } \\
\text { 1960-1970- } \\
\text { 1980, Suecia }\end{array}$ & $\begin{array}{l}\text {-Asbesto } \\
\text {-Polvo de } \\
\text { madera } \\
\text {-Polvo de sílice } \\
\text {-Radiación } \\
\text { ultravioleta } \\
\text {-Tabaco } \\
\text {-Alcohol }\end{array}$ & $\begin{array}{l}n=15 \text { millones } \\
\text { Edad }=30-45 \\
\text { años } \\
\text { SIR } 2.27 \\
\text { H/M=1 }\end{array}$ & $\begin{array}{l}\text { No } \\
\text { consta }\end{array}$ & $\begin{array}{l}\text { Melanoma } \\
\text { Labio } \\
\text { Nariz }\end{array}$ & $\begin{array}{l}\text { - Alta incidencia de cáncer de } \\
\text { labio en pescadores (SIR 2,27, } \\
\text { IC 95\% 2,05- 2,51). } \\
\text { - No asociación significativa } \\
\text { de NMSC (SIR 0,75; IC 95\% } \\
\text { 0,69-0,82). }\end{array}$ \\
\hline $\begin{array}{l}\text { Pukkala } \\
\text { et al } \\
1996(29)\end{array}$ & Cohortes & $\begin{array}{l}\text { 1967-1992, } \\
\text { Finlandia }\end{array}$ & -Radiación UV & $\begin{array}{l}n=42496 \\
H / M=2,68 \\
\text { Edad: }<30 \text { a } \\
\geq 60 \text { años }\end{array}$ & $\begin{array}{l}>20 \\
\text { años }\end{array}$ & $\begin{array}{l}\text { NMSC } \\
\text { (excepto } \\
\text { BCC) } \\
\text { Melanoma }\end{array}$ & $\begin{array}{l}\text { La radiación UV acumulativa } \\
\text { aumenta el riesgo de cáncer } \\
\text { de piel no melanoma }(\mathrm{SIR}=1,8 \text {, } \\
\text { IC } 1,2-2,5)\end{array}$ \\
\hline $\begin{array}{l}\text { Rafnsson } \\
\text { et al, } \\
1995(12)\end{array}$ & Cohortes & $\begin{array}{l}\text { 1958-1986, } \\
\text { Islandia }\end{array}$ & $\begin{array}{l}\text {-Radiación UV } \\
\text {-Asbesto } \\
\text {-Tabaco }\end{array}$ & $\begin{array}{l}\text { Casos n=758 } \\
\text { H/M= no } \\
\text { consta } \\
\text { Edad M: no } \\
\text { consta }\end{array}$ & 10 años & Piel & $\begin{array}{l}\text { No asociación significativa de } \\
\text { NMSC y melanoma (SIR=0,4 } \\
\text { IC } 95 \% \text { 0,13-0,94; SIR=1,51 } \\
\text { IC } 95 \% \text { 0,93-2,3 respectiva- } \\
\text { mente) }\end{array}$ \\
\hline $\begin{array}{l}\text { Svenson } \\
\text { et al, } \\
1995^{(34)}\end{array}$ & Cohortes & $\begin{array}{l}\text { 1965-1988, } \\
\text { Suecia }\end{array}$ & $\begin{array}{l}\text {-PCB } \\
\text {-PCDD } \\
\text {-PCDF } \\
\text {-PUFA } \\
\text {-Radiación UV } \\
\text {-Arsénico }\end{array}$ & $\begin{array}{l}n=11373 \\
\text { H/M=1 } \\
\text { Edad: no } \\
\text { consta. }\end{array}$ & $\geq 1$ año & $\begin{array}{l}\text { Carcinoma } \\
\text { de labio } \\
\text { Carcinoma } \\
\text { piel } \\
\text { Melanoma }\end{array}$ & $\begin{array}{l}\text {-Ca piel: SIR costa oeste 1,12 } \\
\text { (IC 95\% 0,88-1,43), costa este } \\
\text { 2,28 (IC 95\% 1,45-3,5) } \\
\text {-Ca labio: SIR costa oeste 1,92 } \\
\text { (IC 95\% 1,29-2,8), costa este } \\
\text { 2,6 (IC 95\% 1,05-5,36) } \\
\text {-Melanoma: SIR costa oeste } \\
\text { 0,78 (IC 95\% 0,48-1,22), costa } \\
\text { este } 0 \text { (IC 95\% 0-0,72). }\end{array}$ \\
\hline
\end{tabular}




\begin{tabular}{|c|c|c|c|c|c|c|c|}
\hline Autor & $\begin{array}{l}\text { Tipo de } \\
\text { estudio }\end{array}$ & Periodo y país & $\begin{array}{l}\text { Agente(s) } \\
\text { (exposición) }\end{array}$ & $\begin{array}{l}\text { Población } \\
\text { (trabajadores) }\end{array}$ & $\begin{array}{l}\text { Tiempo } \\
\text { exposi- } \\
\text { ción }\end{array}$ & $\begin{array}{l}\text { Efecto } \\
\text { estudiado } \\
\text { (neopla- } \\
\text { sia) }\end{array}$ & Resultados principales \\
\hline $\begin{array}{l}\text { Hagmar } \\
\text { et al, } \\
1992^{(13)}\end{array}$ & Cohortes & $\begin{array}{l}1 \text { Enero 1944- } \\
31 \text { Diciembre } \\
\text { 1987, Suecia } \\
\text { *excluidos } \\
\text { fallecidos antes } \\
\text { de } 1968\end{array}$ & $\begin{array}{l}\text {-Radiación solar } \\
\text { UV-B } \\
\text {-Aceites } \\
\text {-Alquitrán } \\
\text {-Arsénico }\end{array}$ & $\begin{array}{l}n=1360 \\
\mathrm{H} / \mathrm{M}=1 / 0 \\
\text { Todas las } \\
\text { edades }\end{array}$ & $\begin{array}{l}\geq 12 \\
\text { meses }\end{array}$ & $\begin{array}{l}\text { Carcinoma } \\
\text { escamoce- } \\
\text { lular } \\
\text { Carcinoma } \\
\text { de labio } \\
\text { Melanoma }\end{array}$ & $\begin{array}{l}\text { Tasa de morbilidad: } \\
\text {-SCC: SMR 2,79 (95\% Cl1,73- } \\
\text { 4,36) } \\
\text {-Ca labio: SMR 3,16 (95\% Cl } \\
\text { 1,16-6,88) } \\
\text {-No se encontraron casos de } \\
\text { melanoma }\end{array}$ \\
\hline $\begin{array}{l}\text { Vitasa et } \\
\text { al, 1990(14) }\end{array}$ & $\begin{array}{l}\text { Epide- } \\
\text { miológico } \\
\text { transversal }\end{array}$ & $\begin{array}{l}\text { Marzo } \\
\text { 1985-Enero } \\
\text { 1986, EEUU }\end{array}$ & $\begin{array}{l}\text {-Radiación solar } \\
\text { UV-B }\end{array}$ & $\begin{array}{l}n=808 \\
H / M=1 / 0 \\
\text { Edad: } \geq 30 a\end{array}$ & $\begin{array}{l}\text { Desde } \\
\text { que el } \\
\text { indi- } \\
\text { viduo } \\
\text { tiene } 15 \\
\text { años }\end{array}$ & $\begin{array}{l}\text { SCC } \\
\text { BCC } \\
\text { AK }\end{array}$ & $\begin{array}{l}\text { Para aquellos sujetos cuya ex- } \\
\text { posición superó el tercer cuartil, } \\
\text { el RR de SCC aumentó x2,5, } \\
\text { mientras que no tuvo mayor } \\
\text { riego de BCC o AK }\end{array}$ \\
\hline $\begin{array}{l}\text { Whitaker } \\
\text { et al, } \\
1979(36)\end{array}$ & Cohortes & $\begin{array}{l}\text { 1967-1969, } \\
\text { England }\end{array}$ & $\begin{array}{l}\text {-Radiación UV } \\
\text {-Tabaco }\end{array}$ & $\begin{array}{l}n=781 \\
\text { H/M=1,5 } \\
\text { Edad: } \geq 15 \\
\text { años }\end{array}$ & 20 años & SCC & $\begin{array}{l}\text { Los resultados de este estudio } \\
\text { carecen de significación } \\
\text { estadística para la profesión } \\
\text { pesquera, puesto que solo se } \\
\text { incluyeron dos pescadores en } \\
\text { la muestra }\end{array}$ \\
\hline $\begin{array}{l}\text { Spitzer et } \\
\text { al, 1975(16) }\end{array}$ & $\begin{array}{l}\text { Caso-Con- } \\
\text { trol y de } \\
\text { cohortes }\end{array}$ & $\begin{array}{l}\text { 1961-1971, } \\
\text { Canadá }\end{array}$ & $\begin{array}{l}\text {-Pesca } \\
\text {-Exterior } \\
\text {-Fumar en pipa }\end{array}$ & $\begin{array}{l}n=576 \\
\text { H/M=1/0. } \\
\text { Edad: } 35-69 \\
\text { años }\end{array}$ & $\geq 8$ años & $\begin{array}{l}\text { Carcinoma } \\
\text { de labio } \\
\text { Carcinoma } \\
\text { de piel en } \\
\text { la cabeza y } \\
\text { el cuello }\end{array}$ & $\begin{array}{l}\text { Ca piel RR 0,78 (no significa- } \\
\text { tivo). } \\
\text { Ca labio RR= 1,65; } p<0,01 \text {. }\end{array}$ \\
\hline
\end{tabular}

AK= Queratosis actínica; BCC = Carcinoma basocelular; NMSC = Cáncer de piel tipo no melanoma; H/M= proporción hombres/mujeres; PCB=bifenilos policlorados; $\mathrm{PCDD}=$ dibenzo-p-dioxinas policloradas; PCDF= dibenzofuranos policlorados; PUFA= ácidos grasos n-3 poliinsaturados; RR= riesgo relativo; SCC = Carcinoma espinocelular; SIR= Ratio de incidencia estandarizado; UV= ultravioleta

Tabla 3: Evaluación de la calidad metodológica de los estudios a través de la guía STROBE ${ }^{(5)}$.

\section{Puntuación de los 22 ítems}

\section{$\begin{array}{lllllllllllllllllllllllll}\text { Referencias } & 1 & 2 & 3 & 4 & 5 & 6 & 7 & 8 & 9 & 10 & 11 & 12 & 13 & 14 & 15 & 16 & 17 & 18 & 19 & 20 & 21 & 22 & \text { Total } & \%\end{array}$}

\begin{tabular}{|c|c|c|c|c|c|c|c|c|c|c|c|c|c|c|c|c|c|c|c|c|c|c|c|c|}
\hline \multirow{4}{*}{$\begin{array}{l}\text { Larese et al(1) } \\
\text { Alfonso et } \\
\text { al(7) } \\
\text { Mikoczy et } \\
\text { al(30) } \\
\text { Pukkala et } \\
\text { al(9) }\end{array}$} & 1 & 1 & 1 & 0 & 7 & 1 & 0 & 1 & 0 & 0 & 1 & 1 & 0,66 & 1 & 1 & 1 & NA & 1 & 1 & 1 & 0 & 0 & 14,66 & 69,8 \\
\hline & 1 & 1 & 1 & 0 & 1 & 1 & 0 & 1 & 0 & 1 & 1 & 1 & 0,66 & 0 & 0 & 1 & NA & 1 & 1 & 1 & 0 & 0 & 13,66 & 65 \\
\hline & 1 & 1 & 1 & 0 & 1 & 1 & 0 & 1 & 0 & 1 & 1 & 1 & 0 & 0 & 1 & 1 & NA & 1 & 1 & 1 & 0 & 1 & 15 & 71,4 \\
\hline & 1 & 1 & 0 & 0 & 1 & 1 & 1 & 1 & 0 & 1 & 1 & 1 & 1 & 1 & 1 & 0,33 & NA & 1 & 1 & 1 & 0 & 1 & 16,33 & 77,8 \\
\hline $\begin{array}{l}\text { Pukkala et } \\
a^{(29)}\end{array}$ & 1 & 1 & 1 & 0 & 1 & 1 & 1 & 1 & 0 & 1 & 0 & 0 & 0,66 & 1 & 1 & 0,66 & NA & 1 & 0 & 1 & 0 & 1 & 14,32 & 68,2 \\
\hline $\begin{array}{l}\text { Rafnsson et } \\
a^{(12)}\end{array}$ & 1 & 0 & 0 & 0 & 1 & 1 & 0 & 1 & 0 & 1 & 0 & 0 & 0,66 & 1 & 1 & 0,66 & NA & 1 & 1 & 1 & 0 & 1 & 12,32 & 58,7 \\
\hline $\begin{array}{l}\text { Svenson et } \\
a^{(34)}\end{array}$ & 1 & 1 & 1 & 0 & 1 & 1 & 0 & 1 & 0 & 1 & 1 & 1 & 0 & 0 & 1 & 1 & NA & 1 & 0 & 1 & 0 & 1 & 14 & 66,7 \\
\hline $\begin{array}{l}\text { Hagmar et } \\
\text { al(13) }\end{array}$ & 0,5 & 1 & 1 & 0 & 1 & 1 & 1 & 1 & 0 & 1 & 1 & 1 & 0 & 0 & 1 & 1 & NA & 1 & 1 & 1 & 0 & 1 & 15,5 & 73,8 \\
\hline $\begin{array}{l}\text { Vitasa et } \\
\text { al(14) }\end{array}$ & 1 & 1 & 1 & & 0 & 1 & 0 & 0 & 0 & 0 & 0 & 1 & 0,33 & 1 & 1 & 1 & NA & 1 & 1 & 1 & 0 & 1 & 12,33 & 58,7 \\
\hline $\begin{array}{l}\text { Whitaker et } \\
\text { al }^{(36)}\end{array}$ & 1 & 1 & 0 & D & 1 & 1 & 0 & 1 & 0 & 1 & 1 & 0,33 & 0,66 & 1 & 1 & 1 & NA & 1 & 1 & 1 & 0 & 1 & 14,99 & 71,3 \\
\hline $\begin{array}{l}\text { Spitzer et } \\
a l^{(16)}\end{array}$ & 1 & 1 & 1 & & 1 & 1 & 0 & 0 & 1 & 1 & 1 & 0 & 0 & 1 & 1 & 1 & NA & 0 & 1 & 1 & 1 & 1 & 15 & 71,4 \\
\hline
\end{tabular}




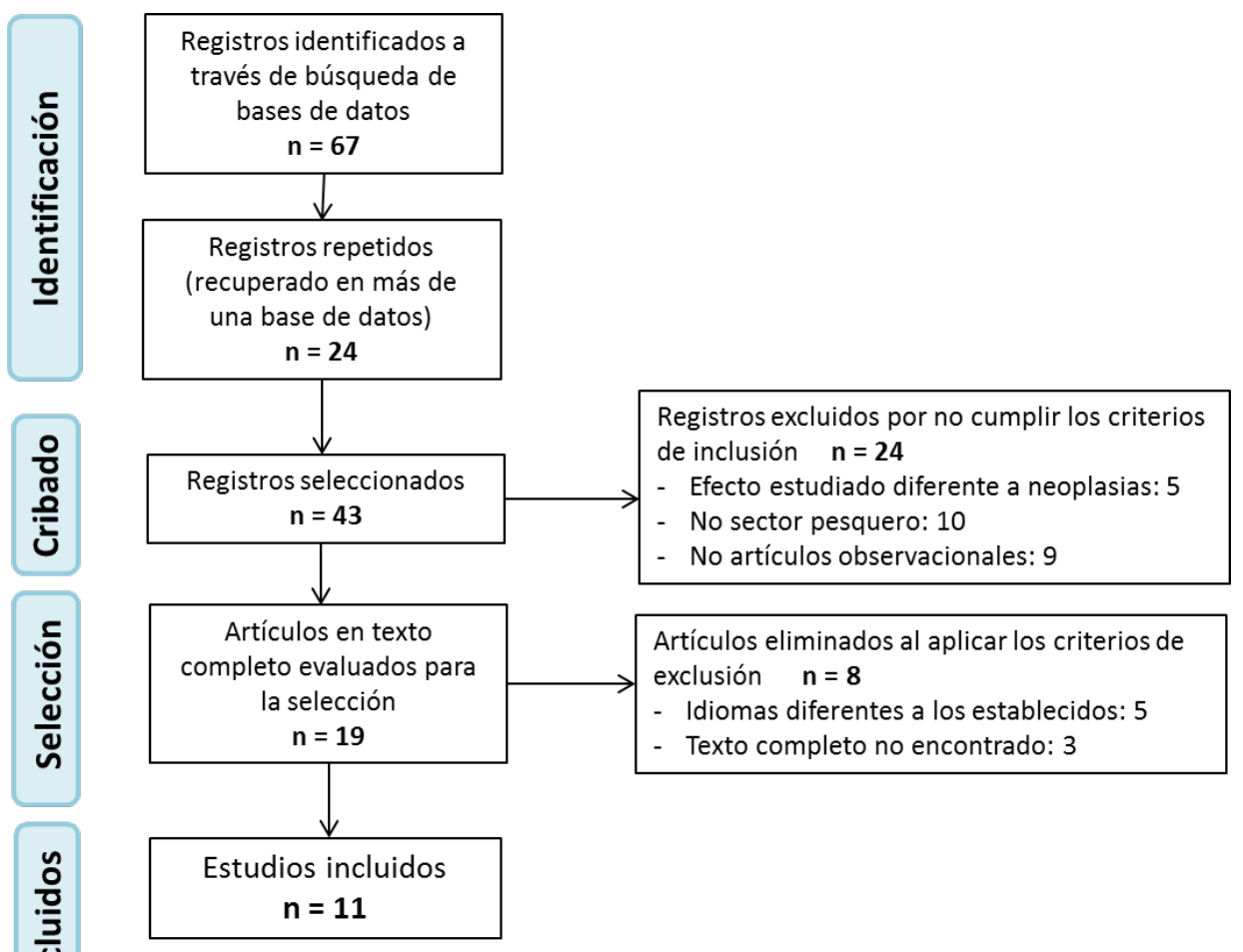

Figura 1: Identificación y selección de estudios.

\section{Limitaciones del estudio}

Los resultados de esta revisión están limitados por las carencias de cada trabajo incluido en la misma(46). Según la US Agency for Health Research and Quality, los diseños epidemiológicos de los estudios seleccionados en esta revisión (cohortes, casos y controles), aportan un nivel de evidencia y grado de recomendación IIb y III, no pudiendo asegurar por completo la validez y fiabilidad de las observaciones realizadas ${ }^{(47,48)}$. Además, la mayoría de estudios no especificaron si realizaron control de los factores de confusión que pudieran afectar a los resultados. También se han encontrado dificultades para dilucidar si incluyeron mediciones cuantitativas de la exposición a la radiación ultravioleta y/o a los otros factores (tabaco y asbesto, entre otros). Al mismo tiempo, resulta difícil valorar el riesgo de neoplasias en la piel en mujeres, dado que no estuvieron representadas en todos los estudios.

Otra limitación ha sido la escasez de artículos encontrados y la poca actualidad de los mismos, siendo la mayoría de ellos publicados en los años 90, aunque sí se ha observado un aumento del interés por el tema en los últimos tres años ${ }^{(1,7)}$. Quizá, este bajo número de trabajos localizados podría indicar que la ecuación de búsqueda fue muy específica, surgiendo la duda de un posible silencio documen- 
tal. Ahora bien, la búsqueda manual en los listados bibliográficos de los artículos incluidos no aportó nuevos documentos a incluir en la revisión.

\section{Conclusiones y perspectivas}

Teniendo en cuenta que las personas permanecen de media un tercio de su vida en su lugar de trabajo(46), en las ocupaciones expuestas a estos agentes se deben siempre aplicar las medidas preventivas adecuadas con el fin de minimizar dicha exposición y por consiguiente disminuir el número de patologías asociadas a la misma. La exposición excesiva a radiación UV, como se ha demostrado en esta revisión, es la primera causa de neoplasias cutáneas en el mundo(1,2), siendo el sector pesquero uno de los más afectos por este y otros factores que pueden producir alteraciones de diferente gravedad en la salud de las personas.Para evitar los efectos nocivos debidos a la exposición a la radiación solar, así como a otros agentes de riesgo para el desarrollo de cáncer de piel, deberían emplearse equipos de protección, tales como guantes, casco o crema protectora ${ }^{(1)}$. Sin embargo, su uso no está tan extendido como cabría esperar en profesiones con alto riesgo de desarrollo de patología cutánea. Por ello, la implantación de medidas preventivas enfocadas al adecuado uso de estos equipos debería ser prioritaria entre los trabajadores del sector de la pesca y en toda profesión con altos niveles de exposición a factores predisponentes al desarrollo de neoplasias malignas en la piel.

A pesar de haberse encontrado artículos que resultaron significativos en cuanto a la vinculación entre las lesiones malignas y la exposición a la radiación ultravioleta en el ámbito laboral, serían necesarios más estudios con diseños mas específicos que aporten evidencia acerca de la relación causal de las neoplasias de piel y el hecho de trabajar al aire libre.

\section{Financiación}

Este trabajo no ha contado con ningún tipo de financiación.

\section{Conflicto de intereses}

Las autoras y el autor de la presente revisión declaran la no existencia de conflicto de interés.

\section{Bibliografía}

1. Larese Filon F, Buric M, Fluehler C. UV exposure, preventive habits, risk perception, and occupation in NMSC patients: A case-control study in Trieste (NE Italy). Photodermatol Photoimmunol Photomed. 2019;35:24-30.

2. Burke WA, Griffith DC, Scott CM, Howell ER. Skin problems related to the occupation of commercial fishing in North Carolina. N C Med J. 2006;67:260-5. 
3. Modenese A, Ruggieri FP, Bisegna F, Borra M, Burattini C, Della Vecchia E, et al. Occupational Exposure to Solar UV Radiation of a Group of Fishermen Working in the Italian North Adriatic Sea. Int J Environ Res Public Health. 2019;16:e3001.

4. Troncoso-Piñeiro P, González-de Giarratana AE, Rivadulla-Lema I, Torres-Romero MG, Sanz-Valero J. Neoplasias en trabajadores expuestos al aluminio y/o sus compuestos: Revisión sistemática. Med Segur Trab. 2018;64:312-26.

5. Von Elm E, Altman D, Egger M, Pocock SJ, Gøtzsche PC, Vandenbroucke JP; Iniciativa STROBE. Declaración de la Iniciativa STROBE (Strengthening the Reporting of Observational studies in Epidemiology): directrices para la comunicación de estudios observacionales. Gac Sanit. 2008;22:144-50.

6. Rocholl M, Ludewig M, Skudlik C, Wilke A. Occupational skin cancer : Prevention and recommendations for UV protection as part of the treatment approved by the public statutory employers' liability insurance. Hautarzt. 2018;69:462-70.

7. Alfonso JH, Martinsen JI, Pukkala E, Weiderpass E, Tryggvadottir L, Nordby KC, et al. Occupation and relative risk of cutaneous squamous cell carcinoma (CSCC): A 45-year follow-up study in 4 Nordic countries. J Am Acad Dermatol. 2016;75:548-55.

8. Bauer A, Beissert $S$, Knuschke P. Prevention of occupational solar UV radiation-induced epithelial skin cancer. Hautarzt. 2015;66:173-8.

9. Pukkala E, Martinsen JI, Lynge E, Gunnarsdottir HK, Sparén P, Tryggvadottir L, Occupation and cancer - follow-up of 15 million people in five Nordic countries. Acta Oncol Stockh Swed. 2009;48:646-790.

10. Gruber F, Peharda V, Kastelan M, Brajac I. Occupational skin diseases caused by UV radiation. Acta Dermatovenerol Croat. 2007;15:191-8.

11. Hannuksela-Svahn A, Pukkala E, Karvonen J. Basal cell skin carcinoma and other nonmelanoma skin cancers in Finland from 1956 through 1995. Arch Dermatol. 1999;135:781-6.

12. Rafnsson V, Gunnarsdóttir $H$. Cancer incidence among seamen in Iceland. Am J Ind Med. 1995;27:187-93.

13. Hagmar L, Lindén $K$, Nilsson A, Norrving B, Akesson B, Schütz A, et al. Cancer incidence and mortality among Swedish Baltic Sea fishermen. Scand J Work Environ Health. 1992;18:217-24.

14. Vitasa BC, Taylor HR, Strickland PT, Rosenthal FS, West $S$, Abbey H, et al. Association of nonmelanoma skin cancer and actinic keratosis with cumulative solar ultraviolet exposure in Maryland watermen. Cancer. 1990;65:2811-7.

15. Chambers LW, Spitzer WO. A method of estimating risk for occupational factors using multiple data sources: the Newfoundland lip cancer study. Am J Public Health. 1977;67:176-9.

16. Spitzer WO, Hill GB, Chambers LW, Helliwell BE, Murphy HB. The occupation of fishing as a risk factor in cancer of the lip. N Engl J Med. 1975;293:419-24. 
17. Balus L, Homoriceanu D. Research on the epidemiology of cutaneous epitheliomas and senile actinic keratoses among a community of fishermen in the Danube delta (Rumania). Arch Belg Dermatol Syphiligr. 1971;27:329-38.

18. Jensen O, Flores A, Bygvraa DA, Baygi F, Charalambous G. A Review of Epidemiological Studies in Latin American Fishing. J Agromedicine. 2019;24:341-50.

19. Bernardes Filho F, de Oliveira Alves A, Towersey L, Hay R, Montag A, Coutinho, Lupi O, et al. The skin health of fishermen in Guanabara Bay, Rio de Janeiro, Brazil. Int J Dermatol. 2019;58:483-90.

20. Gangemi S, Miozzi E, Teodoro M, Briguglio G, De Luca A, Alibrando C, et al. Occupational exposure to pesticides as a possible risk factor for the development of chronic diseases in humans. Mol Med Rep. 2016;14:4475-88.

21. Schwantes H, Dumke AK, Blome O, Elsner P. Actinic keratosis and squamous cell carcinoma- Occupational aspects. Aktuelle Dermatol. 2012;38: 287-93.

22. Ramirez CC, Federman DG, Kirsner RS. Skin cancer as an occupational disease: The effect of ultraviolet and other forms of radiation. Internati Onal J Dermatol. 2005;44:95-100.

23. Harries MJ, Lear JT. Occupational Skin Infections. Occup Med. 2004;54: 441-9.

24. Camacho FM. Chronic radiation dermatitis: What's new in management? J Eur Acad Dermatol Venereol. 2000;14:246-7.

25. De Morais RM, Nogueira Diógenes MJ, Veras OBV. Occupational contact dermatitis in fishermen of Cumbuco beach, Ceara, northeast Brazil. Contact Dermatitis. 2000;42:277-8.

26. Lasanta J, Cabre J. Verrucomas en trabajadores en la industria del pescado. Actas Dermo-Sifiliográficas. 1968;59:305-8.

27. Oldenburg M, Kuechmeister B, Ohnemus U, Baur X, Moll I. Actinic keratosis among seafarers. Arch Dermatol Res. 2013;305:787-96.

28. Oldenburg $M$, Harth $V$, Manuwald U. Hospitalization due to cancer among German seafarers. Am J Ind Med. 2015;58:456-63.

29. Pukkala E, Saarni H. Cancer incidence among Finnish seafarers, 1967-92. Cancer Causes Control. 1996; 7: 231-9.

30. Mikoczy Z, Rylander L. Mortality and cancer incidence in cohorts of Swedish fishermen and fishermen's wives: Updated findings. Chemosphere. 2009;74:938-43.

31. Popim RC, Corrente JE, Marino JAG, Arantes de Souza C. Skin cancer: Use of preventive measures and demographic profile of a risk group in the city of Botucatu. Cienc Saude Colet. 2008;13:1331-6.

32. Diepgen TL, Blome O. UV-light induced skin cancer - A new occupational disease. Dermatol Beruf Umw. 2008;56:47-56.

33. Chobanova N, Vukov M, Yagova A. Cancer incidence among Bulgarian medical radiation workers: Epidemiological study. J BUON. 2007;12:65-9. 
34. Svensson B-G, Mikoczy Z, Strömberg U, Hagmar L. Mortality and cancer incidence among Swedish fishermen with a high dietary intake of persistent organochlorine compounds. Scand J Work Environ Health. 1995;21:106-15.

35. Magnani C, Coggon D, Osmond C, Acheson ED. Occupation and five cancers: a case-control study using death certificates. Br J Ind Med. 1987;44:769-76.

36. Whitaker CJ, Lee WR, Downes JE. Squamous cell skin cancer in the North-west of England, 1967-69, and its relation to occupation. Br J Ind Med. 1979;36:43-51.

37. Cabre J, Lasanta J. Malignant epithelial tumors in the seashore fisherman. Actas Dermosifiliogr. 1968;59:361-4.

38. Lichte V, Dennenmoser B, Dietz K, Häfner HM, Schlagenhauff B, Garbe C, et al. Professional risk for skin cancer development in male mountain guides - a crosssectional study. J Eur Acad Dermatol Venereol. 2010;24:797-804.

39. Thieden E, Collins SM, Philipsen PA, Murphy GM, Wulf HC. Ultraviolet exposure patterns of Irish and Danish gardeners during work and leisure. $\mathrm{Br} J$ Dermatol 2005;153:795-801.

40. Moehrle M, Dennenmoser B, Garbe C. Continuous long-term monitoring of UV radiation in professional mountain guides reveals extremely high exposure. Int $\mathrm{J}$ Cancer. 2003;103:775-8.

41. Moehrle M, Korn M, Garbe $C$. Bacillus subtilis spare film dosimeters in personal dosimetry for occupational solar ultraviolet exposure. Int Arch Occup Environ Health. 2000;73:575-80.

42. Fowler J. Occupational dermatology. Curr Probl Dermatol. 1998;10(6): 211-44.

43. Zaldivar R, Prunes $L$, Ghai G. Arsenic Dose in Patients with Cutaneous Carcinomata and Hepatic Haemangio- Endothelioma after Environmental and Occupational Exposure. Arch Toxicol. 1981;47:145-54.

44. Álvarez Velásquez S, Sanz Valero J. Ventajas de la quimioterapia domiciliaria en los enfermos adultos con neoplasias: revisión sistemática. Hosp Domic. 2020;4:25-41.

45. Epstein S. Environmental Determinants of Human Cancer. Cancer Res. 1974;34:2425-35.

46. Domingo-Pueyo A, Sanz-Valero J, Wanden-Berghe C. Efectos sobre la salud de la exposición laboral al cromo y sus compuestos: revisión sistemática. Arch Prev Riesgos Labor 2014;17:142-53.

47. U.S. Agency for Healthcare Research and Quality. Clinical Guidelines and Recommendations [homepage]. Rockville, USA: Agency for Healthcare Research and Quality [updated 2003 Jul 26; cited 26 Sep 2003]. Disponible en: https://www.ahrq. gov/prevention/guidelines/index.html

48. Wanden-Berghe C, Sanz-Valero J. Systematic reviews in nutrition: standardized methodology. Br J Nutr. 2012; 107 Suppl 2:S3-7. 